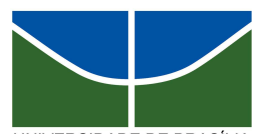

UNIVERSIDADE DE BRASÍLIA

Centro de Excelência em Turismo

Pós-graduação Lato Sensu

Curso de Especialização em Gastronomia e Saúde

\title{
REDUÇÃO DO PALADAR EM IDOSOS PARA OS GOSTOS DOCE E SALGADO
}

\author{
Joana Holanda Cavalcanti Correia
}

Orientadora: Professora MSc. Lívia de Lacerda de Oliveira Pineli

Brasília - 2008 


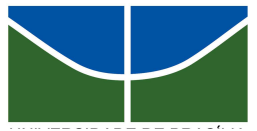

UNIVERSIDADE DE BRASÍLIA

Centro de Excelência em Turismo

Pós-graduação Lato Sensu

Curso de Especialização em Gastronomia e Saúde

\title{
REDUÇÃO DO PALADAR EM IDOSOS PARA OS GOSTOS DOCE E SALGADO
}

\author{
Joana Holanda Cavalcanti Correia
}

Orientadora: Professora MSc. Lívia de Lacerda de Oliveira Pineli

Monografia apresentada ao Centro de Excelência em Turismo - CET, da Universidade de Brasília UnB, como requisito parcial à obtenção do grau de Especialista em Gastronomia e Saúde.

Brasília - 2008 


\section{UNIVERSIDADE DE BRASÍLIA}

Centro de Excelência em Turismo

Pós-graduação Lato Sensu

Curso de Especialização em Gastronomia e Saúde

Monografia apresentada ao Centro de Excelência em Turismo - CET, da Universidade de Brasília - UnB, como requisito parcial à obtenção de grau de Especialista em Gastronomia e Saúde.

\section{REDUÇÃO DO PALADAR EM IDOSOS PARA OS GOSTOS DOCE E SALGADO \\ Joana Holanda Cavalcanti Correia}

Aprovado por:

Prof. Msc. Lívia de Lacerda de Oliveira Pineli

Prof. Dra. Raquel Braz Assunção Botelho

Prof. Msc. Renata Puppin Zandonadi

Brasília, 08 de Agosto de 2008 


\section{DEDICATÓRIA}

Dedico em primeiro lugar a Deus, pois sem a força divina nada é possível, dedico ainda aos meus pais por me proporcionarem mais uma vez essa oportunidade e por acreditarem em mim e por fim dedico este trabalho a todos os idosos que participaram direta ou indiretamente dessa pesquisa. 
Agradeço a professora Lívia pela paciência e orientação. A Anita Martins, Vanessa Cardoso, Marcos Sodré, Glícia e Aldalívia, por colaborarem na elaboração dos testes. A instituição, por participar e ceder o local da realização da pesquisa, aos idosos e aos adultos que participaram do experimento e por fim, ao Vitor Borges, por colaborar para realização deste trabalho e por me dar apoio em todos os momentos. 


\section{LISTA DE ILUSTRAÇÕES}

Figura 1. Ficha de resposta do teste triangular para os gostos doce e

salgado

Figura 2. Ficha de escala hedônica mista para as preparações de sopa e

suco

Figura 3. Medidas de aceitação de refrescos de caju com diferentes

concentrações de açúcar

Figura 4. Medidas de aceitação de sopas com diferentes concentrações de sal 
SUMÁRIO

RESUMO

ABSTRACT

INTRODUÇÃO

METODOLOGIA

RESULTADOS E DISCUSSÃO

CONCLUSÃO

REFERÊNCIAS BIBLIOGRÁFICAS

ANEXOS
08

09

09

12

14

16

17

19

7 


\title{
Revista de Nutrição
}

\section{Redução do paladar em idosos, para os gostos doce e salgado.}

\author{
Reduction of sweet and salt tastes in elderly.
}

\section{Joana Holanda Cavalcanti Correia; Lívia de Lacerda de Oliveira Pineli}

\author{
IUniversidade de Brasília, Centro de Excelência em turismo, \\ Programa de Pós-Graduação.Brasília, DF, Brasil.
}

\section{RESUMO}

INTRODUCAO: Um quadro de anorexia, é muito comum nos idosos, e um dos principais fatores é a alteração no olfato e gustação. OBJETIVO: Verificar o grau de redução do paladar em idosos institucionalizados em comparação ao paladar de adultos e se esta redução afeta a aceitabilidade de uma preparação doce e uma salgada. MÉTODOS: A amostra foi composta por 30 idosos entre 60 a 80 anos, de ambos os sexos, moradores de uma instituição pública e 50 adultos entre 20 a 49 anos, de ambos os sexos, freqüentadores da Universidade Católica de Brasília . Foi determinado o índice limiar de detecção de gostos doce e salgado para os adultos e idosos utilizando-se o método de estímulo constante. Em seguida os idosos participaram de teste de aceitação com escala hedônica para avaliação de refresco e de sopa com diferentes concentrações de açúcar e sal, respectivamente. RESULTADOS: Constatou-se que os idosos avaliados precisaram de aproximadamente nove vezes mais açúcar que os adultos para detectar a presença deste componente, e de aproximadamente vinte e cinco vezes mais sal $(\mathrm{NaCl})$ do que os adultos para detectar a presença desta substância. Foi possível constatar que quanto menor a concentração de açúcar e de sal, maior foi a aceitação da preparação. Isso provavelmente se justifica por efeito de hábito alimentar. CONCLUSÃO: Ao se comparar o limiar do paladar dos idosos ao dos adultos observou-se a diminuição previamente relatada. $O$ uso de medicamentos e a presença de patologias, podem contribuir para tais resultados.

Termos de indexação: Idosos, paladar, funções sensoriais, doce, salgado, limiar de detecção.

\section{ABSTRACT}

INTRODUCTION: A picture of anorexia, is very common in the elderly, and one of the main factors is the change in smell and taste. OBJECTIV: Check the degree of 
reduction of taste in institutionalized elderly in comparison to the taste of adults and whether this reduction affects the acceptability of a fresh preparation and a salt. METHODS: The sample was composed of 30 elderly people between 60 to 80 years, of both sexes, residents of a public institution and 50 adults from 20 to 49 years, of both sexes, visitors at the Catholic University of Brasilia. It was determined the index detection threshold of sweet and salty tastes for adults and older people using the method of constant stimulus. Then the elderly participated in test of acceptance with scale hedônic for evaluation of soft drink and soup with different concentrations of sugar and salt, respectively. RESULTS: It was assessed that the elderly needed approximately nine times more sugar as adults to detect the presence of this component, and approximately twenty-five times more salt $(\mathrm{NaCl})$ than adults to detect the presence of this substance. It was noted that the lower the concentration of sugar and salt, was the greater acceptance of preparation. This probably is justified by the effect of feeding habits. CONCLUSION: When you compare the threshold of taste to the elderly of adults there was a decline previously reported. The use of drugs and the presence of diseases, can contribute to such results.

Terms of indexing: Elderly, taste, sensory functions, sweet, salty, threshold of detection.

\section{INTRODUÇÃO}

O envelhecimento é processo natural, que tem início na concepção e termina na morte. Durante o período de crescimento, os processos anabólicos excedem as alterações catabólicas predominando o aumento do numero de células. Uma vez que o organismo atinja a maturidade fisiológica, a taxa de alteração catabólica ou degenerativa se torna maior que a taxa de regeneração celular anabólica. A resultante perda de células leva a vários graus de eficiência diminuída e de função orgânica prejudicada. ${ }^{1}$

Assim, o envelhecimento, apesar de ser um processo natural, submete 0 organismo a diversas alterações anatômicas e funcionais, com repercussões nas condições de saúde e nutrição do idoso. Muitas dessas mudanças são progressivas, ocasionando efetivas reduções na capacidade funcional, desde a sensibilidade para os gostos primários até os processos metabólicos do organismo. ${ }^{2}$

Para correção das alterações decorrentes do envelhecimento, é freqüente o uso concomitante de múltiplos medicamentos que influenciam na ingestão de alimentos, na digestão, na absorção e na utilização de diversos nutrientes, o que pode comprometer o estado de saúde e as necessidades nutricionais do indivíduo idoso. ${ }^{3}$

No Brasil, estima-se que em 2022 o grupo populacional dos idosos apresentará um crescimento considerado, assim como este fenômeno em nível mundial. Nos próximos 20 anos as estimativas do Brasil são de que a população 
idosa irá exceder 30 milhões de pessoas, o que pode chegar a representar quase $13 \%$ da população. ${ }^{4}$

O envelhecimento populacional brasileiro surge acompanhado de uma transição social, apresentando famílias menores e com necessidades financeiras que exigem a participação das mulheres no mercado de trabalho, reduzindo para muitos idosos, a perspectiva de envelhecimento em um ambiente familiar seguro. ${ }^{5}$

A senilidade geralmente promove mais do que a perda de células e funções por si só. Um quadro de anorexia fisiológica multicausal é muito comum nos idosos em geral. Os principais fatores correlacionados a esta anorexia são: isolamento social, alterações no sistema gastrintestinal vinculado a saciedade, elevação da leptina nos homens decorrente da redução de testosterona, saúde bucal precária e alteração no olfato e gustação. ${ }^{6}$ As disfunções de paladar e olfato tendem a começar aos 60 anos de idade e podem se tornar mais graves nas pessoas acima de $70{ }^{7}$

De acordo com a natureza do estímulo que são capazes de captar, os receptores sensoriais podem ser classificados em: quimiorreceptores, que detectam substâncias químicas, por exemplo, na língua e nas narinas, responsáveis pelos sentidos do paladar e do olfato; termorreceptores, que captam estímulos de natureza térmica, distribuídos por toda a pele e mais concentrado nas regiões da face, dos pés e das mãos; mecanorreceptores, que captam estímulos mecânicos, no ouvido interno, por exemplo, capazes de captar ondas sonoras e que também funcionam como órgãos de equilíbrio e, por fim, os que captam estímulos luminosos, como nos olhos. $^{8}$

O ser humano, assim como outros animais, possui sistemas sensoriais especiais, que incluem o sistema visual, auditivo, vestibular, olfativo e gustativo. Estes sistemas sensoriais permitem a detecção e análise da luz, do som, dos sinais químicos, bem como a sinalização da posição da cabeça do indivíduo. ${ }^{9}$ As sensações gustativas do doce, do azedo, do amargo, do salgado, são criadas por esta tradução de sinais químicos liberados pelo alimento. ${ }^{8}$

As alterações sensoriais da audição, olfato e sensação do gosto estão associadas à diminuição de apetite no idoso, comprometendo o seu comportamento alimentar e estado nutricional. A visão é um dos fatores que também levam a diminuição do apetite do idoso, a qual é responsável pelo reconhecimento dos alimentos. Porém, o que mais interfere, é a alteração do olfato e da gustação, nos quais a diminuição dos receptores gustativos e olfativos interfere no gosto dos alimentos. ${ }^{5}$ Estas alterações podem resultar de uma variedade de fatores, do 
envelhecimento normal, doenças como Alzheimer, medicações, intervenções cirúrgicas, radioterapia e fatores sócio-ambientais. ${ }^{1} \mathrm{~A}$ perda da sensação de paladar e olfato pode não apenas reduzir o prazer e conforto associados aos alimentos, mas também aumentar o perigo de envenenamento alimentar ou exposição em excesso a substâncias químicas prejudiciais, presentes no ambiente que, de outro modo, seriam detectáveis por estes sentidos. ${ }^{10}$

Observa-se um declínio mais acentuado do paladar para os sabores salgado e amargo. Este fato explica a tendência do indivíduo idoso acrescentar sal ao alimento, fator deletério, especialmente para os portadores de doenças cardiovasculares. Além disso, ocorre diminuição do volume de saliva, da amilase e conseqüentemente de sua atividade. Como amilase é responsável pela digestão inicial dos carboidratos, ainda na boca, há perda de capacidade de percepção do gosto doce. ${ }^{11}$

Durante muito tempo pensou-se ser a língua setorizada quanto à percepção gustativa. Inclusive testes neurológicos foram desenvolvidos baseados na falsa premissa que botões gustativos específicos para determinados sabores se concentravam em certas regiões da língua. Esses botões estão dispersos pelo dorso da língua e do palato e se apresentam em menor número em outras regiões. São as células dos botões gustativos responsáveis por se perceber os sabores. $\mathrm{O}$ doce e 0 amargo são percebidos por meio de receptores de membrana, enquanto, em contrapartida, o salgado e o ácido dependem de canais iônicos especializados de Sódio $(\mathrm{Na}+)$ e Hidrogênio $\mathrm{H}+{ }^{12}$

Todos os cinco sentidos são importantes para se desfrutar do alimento. Como o "input" proveniente de um canal sensorial pode interagir com os outros, em ambos os níveis, psicológico e fisiológico, não é possível considerar qualquer um deles separadamente, ao se avaliar qualidade sensorial dos alimentos. ${ }^{13} \mathrm{Cor}$, aroma, temperatura, consistência, estado físico são fatores que isolada ou conjuntamente exaltam características sensoriais e gastronômicas e influenciam os sentidos. ${ }^{14}$

Sabe-se que a diminuição dos sentidos está correlacionada com a perda de peso e que esta última é fator colaborativo no aumento da internação de idosos. ${ }^{6}$

Diante destes conhecimentos este trabalho teve como objetivo verificar o grau de redução do paladar em idosos institucionalizados em comparação ao paladar de adultos e se esta redução afeta a aceitabilidade de uma preparação doce e uma salgada. 


\section{METODOLOGIA}

O trabalho foi conduzido como pesquisa transversal exploratória. O estudo foi realizado em uma instituição pública localizada no Núcleo Bandeirantes- DF e no Laboratório de Técnica Dietética da Universidade Católica de Brasília (UCB). A instituição é uma casa de apoio que atende a 180 idosos entre 60 a 90 anos aproximadamente.

\section{População}

A amostra foi composta por30 idosos entre 60 a 80 anos, de ambos os sexos, moradores da instituição, e 50 adultos entre 20 a 49 anos de ambos os sexos, freqüentadores da UCB. Foi elaborada uma ficha de recrutamento para os adultos participantes e um termo de consentimento livre e esclarecido para os idosos (ANEXOS I e II).

\section{Critérios de exclusão}

Foram excluídos os idosos que tinham hipertensão e diabetes. Também foram excluídos os idosos que não eram lúcidos, devido ao entendimento do teste. Com isso, apenas 30 idosos foram selecionados.

\section{Método I}

Determinação do índice limiar de detecção.

Para determinação do limiar de detecção de gosto doce em adultos e idosos foi aplicado o método de estímulo constante, consistindo de uma seqüência de testes triangulares com concentrações de açúcar crescentes de 0,024\%, 0,048\%, 0,097\%, $0,195 \%, 0,39 \%, 0,78 \%, 1,56 \%, 3,125 \%$. Em cada triângulo, duas amostras eram água pura e a terceira uma das soluções.

Para determinação do limiar de detecção de gosto salgado em adultos e idosos foi realizada uma seqüência de testes triangulares com concentrações de sódio crescente de 0,0015625\%,0,003125\%,0,00635\%,0,0125\%,0,025\%,0,05\%, $0,1 \%, 0,2 \%, 0,4 \%$. Em ambos os testes foram utilizadas a ficha de avaliação apresentada na Figura 1. 


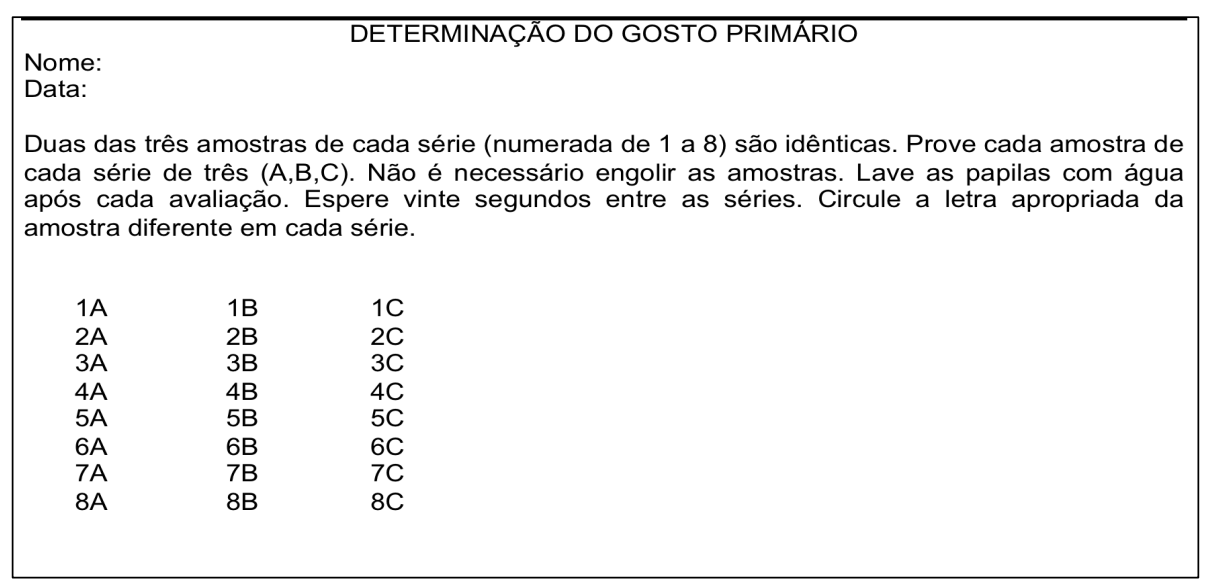

Figura 1. Ficha de resposta do teste triangular para o gosto doce e salgado.

\section{Método II - Testes de Aceitação com escala}

\section{hedônica}

Foi realizado o teste de aceitação com escala hedônica com preparações de refresco de caju e sopa, com os idosos. As formulações avaliadas foram:

Refrescos de caju com concentrações de 3\%, 2,5\%, 2\% e 1\% de açúcar.

Sopas com concentrações de $2 \%, 1 \%, 0,7 \%$ e $0,5 \%$ de sódio.

Refrescos de caju foram preparados por meio da diluição de suco concentrado com água e adição das diferentes concentrações de açúcar. Em seguida, as amostras em garrafas ficaram armazenadas em geladeira por um dia.

A preparação da sopa foi elaborada no Laboratório de Técnica Dietética com os seguintes ingredientes: $140 \mathrm{~g}$ de batata inglesa, $230 \mathrm{~g}$ de chuchu, $70 \mathrm{~g}$ de cebola, $100 \mathrm{~g}$ de abóbora japonesa, $8 \mathrm{~g}$ de alho, $10 \mathrm{~g}$ de mix de temperos: $3 \mathrm{~g}$ de salsa, $3 \mathrm{~g}$ de cebolinha e $5 \mathrm{ml}$ de azeite e diferentes concentrações de sódio. (ANEXO III) As diferentes formulações foram imediatamente engarrafadas e transportadas em isopor até o local do teste.

A avaliação foi realizada com ficha de escala hedônica mista de nove pontos (Figura 2), para melhor adaptação à realidade dos idosos e para melhor entendimento do teste.

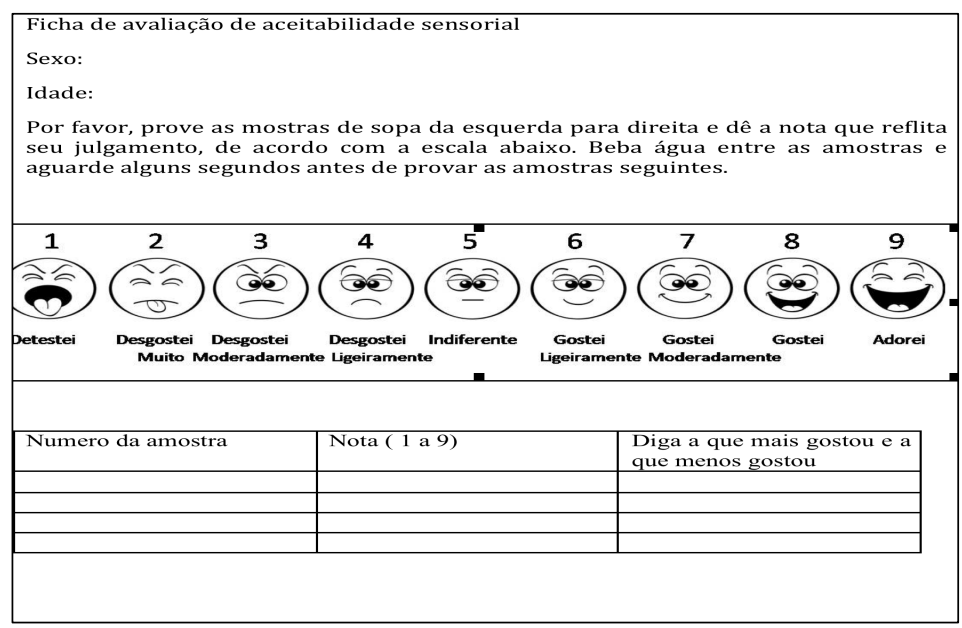


A degustação com os idosos teve inicio às $14 \mathrm{hs}$ e término às $18 \mathrm{hs}$ e $15 \mathrm{~min}$. no primeiro dia. No segundo dia, teve inicio às $14 \mathrm{hs}$ e termino às $17 \mathrm{hs}$ e $30 \mathrm{~min}$. Os idosos tiveram acompanhamento individual durante a aplicação do teste.

\section{Análise de dados}

Método 1: o limiar de detecção individual foi determinado pela concentração correspondente ao crossover, ou seja, concentração antes da qual não havia detecção correta do estímulo e a partir da qual a detecção era sempre correta. O limiar de detecção do grupo foi determinado pela média geométrica dos limiares individuais.

Método 2: o resultado de aceitabilidade das formulações foi determinado por Análise de Variância e teste de comparação da diferença mínima significativa $(p<0,05)$ usando o Programa Statistica 5.0.

\section{RESULTADOS E DISCUSSÃO}

\section{Analise do limiar de detecção do gosto doce}

Pôde-se observar que os adultos avaliados perceberam o gosto doce a partir de $0,114 \%$ de açúcar, enquanto os idosos perceberam o gosto doce a partir de 0,960\% de açúcar, ou seja, o idoso precisou de aproximadamente nove vezes mais açúcar do que o adulto para detectar a presença de açúcar.

A diminuição da sensação do paladar provavelmente é atribuída ao número de corpúsculos (gomas) gustativos em cada papila, que é em torno de 100 no idoso e no adulto chega a ser superior a $250 .^{2}$

Uma substância que não poderá ser detectada em nenhuma circunstância prática possui uma concentração mínima a partir da qual indivíduos com olfato e paladar normais estariam aptos a detectá-la. Este seria o conceito de threshold também utilizado pela associação americana de teste e materiais (The American Society forTestingand Materiais - ASTM). ${ }^{15}$

A saúde oral está diretamente ligada à qualidade de vida do idoso e sua ausência pode comprometer o ato de comer, de saborear os alimentos e até mesmo 
prejudicar os relacionamentos pessoais, por meio de suas funções alteradas da mastigação, da deglutição, do paladar e do olfato. As alterações do olfato e paladar podem contribuir para a desnutrição. ${ }^{13}$

\section{Análise do limiar de detecção do gosto salgado}

Comparando os resultados, pôde-se observar que os adultos perceberam o gosto salgado a partir de $0,010 \%$ de sal $(\mathrm{NaCl})$, enquanto os idosos perceberam o gosto salgado a partir de $0,266 \%$ de sal $(\mathrm{NaCl})$, ou seja o idoso precisou de aproximadamente vinte e cinco vezes mais sal $(\mathrm{NaCl})$ do que o adulto para detectar a presença de sal.

Tais resultados podem justificar o hábito dos idosos de temperarem a comida com mais sal do que o necessário, para que estes possam perceber o sabor. O que possivelmente pode ocasionar um aumento significativo na pressão arterial que geralmente não acontece com pessoas que não tem a alteração dessa percepção. ${ }^{16}$

\section{Análise das preparações de suco e sopa para os idosos}

Com base na figura 3 , foi possível constatar que quanto menor é a concentração de açúcar, maior é a aceitação da preparação. Isso se justifica pelo fato de os idosos serem de uma instituição onde a alimentação deles é hipossódica e reduzida em açúcar, afinal grande parte deles é diabética e hipertensa, com isso o paladar vai se adaptando à realidade. Também na figura 4, observou-se que quanto menor é a concentração de $\mathrm{NaCl}$, maior é a aceitação dos idosos.

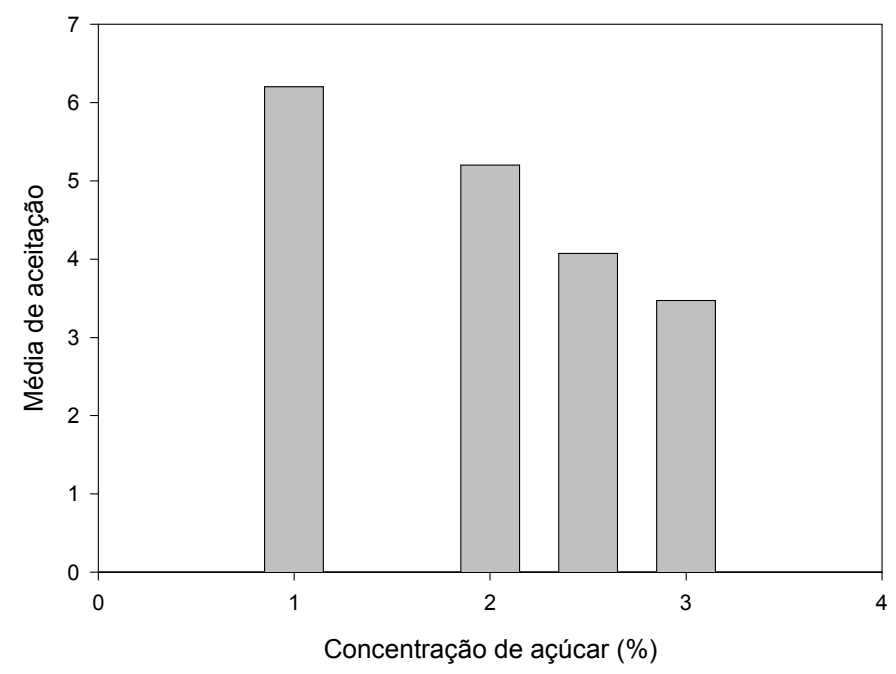

Figura 3: Médias de aceitação de refrescos de caju com diferentes concentrações de açúcar 


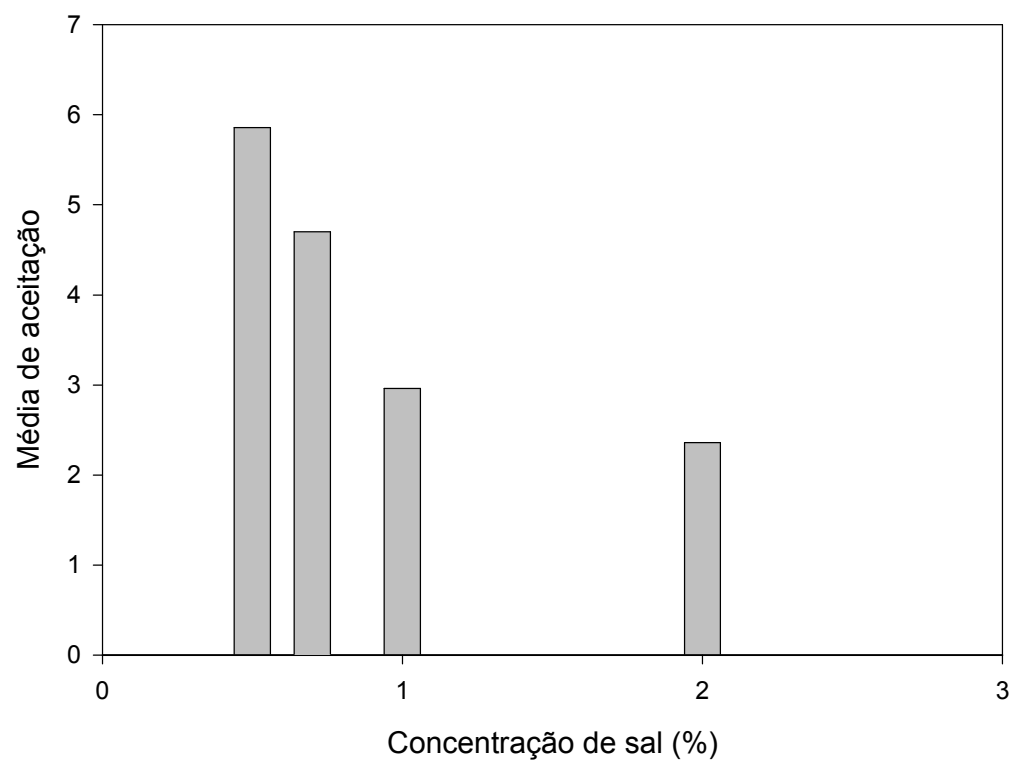

Figura 4. Médias de aceitação de sopas com diferentes concentrações de sal

\section{CONCLUSÃO}

Ao se comparar o limiar de paladar dos idosos ao dos adultos observou-se a diminuição previamente relatada na literatura. O uso de medicamentos e a presença de patologias, correlacionados ao paladar, podem contribuir para tais resultados. $\mathrm{O}$ processo natural de diminuição das funções fisiológicas com a chegada da senilidade é outro fator de grande relevância. Todavia, pôde-se perceber que a redução da percepção não afeta a aceitabilidade. Neste caso, os hábitos alimentares podem ter contribuído para maior aceitação de preparações com menores concentrações de sal e açúcar. Diante de tal complexidade, se fazem necessárias novas pesquisas, para que o conhecimento a respeito da diminuição do paladar com a idade seja ampliado, bem como novas propostas para a reabilitação do idoso a uma alimentação saudável e prazerosa. 


\section{REFERÊNCIAS BIBLIOGRÁFICAS}

1. MAHAN, L. K.; STUMP, S. E. Krause: Alimentos, Nutrição e Dietoterapia. São Paulo: Roca, 2003.

2. CAMPOS, M., T.; MONTEIRO, J., B. ; ORNELAS, A., R., C.; Fatores que afeta o consumo alimentar e a nutrição do idoso. Revista de nutrição, Campinas, v. 13, n. 3, set./ dez., 2000. pp.157-165.

3. ACUÑA, K.; CRUZ, T.Avaliação do Estado Nutricional de Adulto e Idosos e Situação Nutricional da População Brasileira. Arq.BrasEndocrinolMetab vol $48 n^{\circ} 3$ Junho 2004345.

4. PEREIRA, R. S.; CURIONE, C. C. ; VERAS, R. Perfil demográfico da população idosa no Brasil e no Rio de Janeiro em 2002. Textos Envelhecimento v.6 n.1 Rio de Janeiro 2003.

5. SANTElle, O.; LefEVRE, A., C. ; CERVATO, A., M. Alimentação institucionalizada e suas representações sociais entre moradores de instituições de longa permanência para idosos.Caderno Saúde Pública, Rio de Janeiro, v.23, p. 3061-3065, Dez 2007.

6. SHILS, MAURICE E. et al., Modern Nutrition in Health and disease, $10^{\mathrm{a}} \mathrm{ed}$. Philadelphia, Lippincott Williams \& Wilkins, 2006.

7. OLIVEIRA, G., M.; MENEZES, H., S.; O estado nutricional do idoso institucionalizado no lar Moriá. Arquivos Médicos, São Paulo, v. 9, 2006.

8. SANDRINI, Vanessa. Ciências e arte dos sabores. 5a Ed. Caxias do Sul: Editora Educs, 2001.

9. BERNE, ROBERT M. et al. Fisiologia, 5a Ed. São Paulo, Elsevier, 2004.

10. OliveIRA, G., M.; MENEZES, H., S.; O estado nutricional do idoso institucionalizado no lar Moriá. ArquivosMédicos, São Paulo, v. 9, 2006. 
11. YMAUCHI, I; ENDO, S; YOSHIMURA L. A; New Wholemouth gustatory test procedure. II Effects of aging, gender and smoking. ActaOtolaryngolSuppl 2002;(546): 49-59.

12. FABER, Jorge. Avanços na compreensão do paladar. Revista dental Press de Odontologia e Ortopedia facial. V 11, n. 1. Maringá: Jan./fev. 2006.

13. DUTHIE J.; Edmundo H.; KATZ, P. R. Geriatria prática. 3 ed. Rio de Janeiro: Revinter, 2002. 582 p.

14. SILVA-NETO, Cinciano R. MAFFEIS, Eloisa R. Fatores que alteram a percepção gustativa. RevistaFaculdadeOdontologiaLins, v. 3, n.2, PP. 28-32, jul/dez. 1990.

15. LAWLESS, Harry, T; HEYMANN, Hildegarde. Sensory Evaluation of Food.New York: Springer, 1998.

16. BARAJAS, CM; MIRANDA, SM; BARRIENTOS, S. Función gustativa con a respecto a La edad.Univ. Odont. , v. 22, n. 48, p p. 34-40, jun. 2002. 
ANEXOS 


\section{ANEXO I}

\section{- Termos de consentimento}

\section{TERMO DE ConsentIMENTO LIVRE E EsCLARECIDO}

O idoso por quem você é responsável na instituição está sendo convidado a participar, como voluntário, da pesquisa: "Redução do paladar em idosos, para o gosto doce e salgado". No caso de você aceitar que o idoso faça parte do estudo, por favor, assine ao final deste documento, que possui duas vias. Uma delas é sua e a outra do pesquisador responsável. Sua recusa não trará nenhum prejuízo em sua relação com o pesquisador (a) e/ou com a Instituição. Você receberá uma cópia deste termo que consta o telefone e endereço do pesquisador principal, para esclarecimentos de dúvidas sobre o projeto e a participação do idoso. Abaixo estão descritas as informações referentes à pesquisa.

"Redução do paladar em idosos, para o gosto doce e salgado"

Esta pesquisa é um estudo que tem como objetivos realizar a avaliação da redução da sensibilidade do paladar em idosos, com relação aos gostos doce e salgado.

O idoso participará da pesquisa das seguintes formas: Primeiramente apresentam-se oito séries de três amostras (em ordem crescente de concentração): em cada série (triângulo), uma amostra é a solução de sacarose e as outras duas são água à mesma temperatura. Solicita-se ao provador analisar cada série, selecionar a amostra diferente e, se possível, identificar qual o gosto. O mesmo é feito para teste com soluções de sal. Todas as concentrações são muito baixas. Também vai ser feita uma análise usando suco de caju e sopa de hortaliças, serão quatro copos de sopa com concentrações diferentes de sal e o mesmo para o suco com concentrações diferentes de açúcar, serão concentrações muito baixas.

\section{DETERMINAÇÃO DO ÍNDICE LIMIAR DE GOSTO PRIMÁRIO}

Os dados colhidos serão utilizados única e exclusivamente para os objetivos propostos no estudo. As informações são de caráter confidencial, sendo que estas poderão ser publicadas em congressos e revistas científicas, resguardando-se a identificação dos participantes da pesquisa.

A participação do idoso no estudo é voluntária e, mesmo que inicialmente haja o aceite em colaborar, poderá desistir a qualquer momento.

Cabe esclarecer que a identidade do idoso será preservada e que esta pesquisa não Ihe implicará desconfortos, riscos e qualquer despesa.

Em qualquer etapa do estudo, você terá livre acesso a todas as informações coletadas e ao profissional responsável pela pesquisa para esclarecimento de eventuais dúvidas.

O principal pesquisador é a aluna de Pós graduação Joana Holanda, que pode ser encontrada no endereço: QRSW 5 BL A2 Apto 106 Sudoeste, nos telefones: 6184079210 / 61 81559900; e no e-mail: joanacholanda@gmail.com .

Caso haja alguma consideração ou dúvida relacionada à ética desta pesquisa, poderá entrar em contato com o Comitê de Ética em Pesquisa da Universidade de Brasília. Endereço: Campus Universitário Darcy Ribeiro Brasília-DF/ CEP 70910-900/Telefone geral: (5561) 33072022/ email: UnB@unb.br 


\section{TERMO DE CONSENTIMENTO LIVRE E ESCLARECIDO}

Fui suficientemente esclarecido(a) a respeito da Pesquisa "“Redução do paladar em idosos, para os gostos doce e salgado", por meio das informações que li ou que foram lidas para mim, descrevendo o estudo. Ficaram claros para mim quais são os propósitos da pesquisa, os procedimentos a serem realizados e seus desconfortos e riscos, as garantias de confidencialidade, e de esclarecimentos permanentes. Ficou claro também que a participação do idoso é isenta de qualquer despesa.

Concordo voluntariamente que o idoso participe deste estudo, e poderei retirar o meu consentimento a qualquer momento, antes ou durante o mesmo, sem penalidades, prejuízo ou perda de qualquer benefício que eu possa ter adquirido com esta pesquisa.

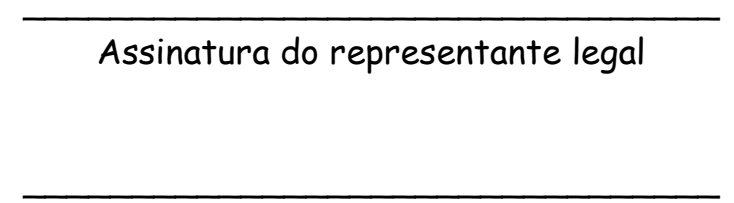

Assinatura da testemunha*

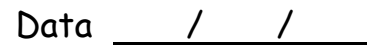

Data

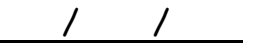

( No caso de participante analfabeto, semi-analfabeto ou portador de deficiência auditiva ou visual).

(Somente para o responsável do projeto):

Declaro que obtive de forma apropriada e voluntária o Consentimento Livre e Esclarecido de da instituição

neste estudo. para a participação

Assinatura do responsável pela pesquisa

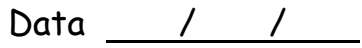


ANEXO II

- Ficha de recrutamento

Ficha de Recrutamento

Pesquisa de Análise Sensorial Redução de Paladar

Nome Completo:

Idade:

Sexo: ( ) Feminino ( ) Masculino

Grau de Escolaridade:

Profissão:

Data de preenchimento:

Como ficou sabendo da pesquisa?

1- Você já participou de alguma pesquisa de análise sensorial ? Caso sim, comete a sua experiência.

2- Você tem algum problema de saúde? Se sim, marque as alternativas a seguir:

Diabetes ( )

Dentição ( )

Hopoglicemia ( )

Hipertensão ( )

Alergias alimentares ( )

Outros ( )

3- Você usa algum medicamento que pode afetar no seu paladar ou olfato?

Hábitos Alimentares

1- Você está fazendo alguma dieta? Qual ?

2- Quantas vezes você como fora por mês?

3- Quantas vezes você come fast-food ao mês?

4- Quantas vezes no mês você come comida congelada? 
5- Qual seu alimento preferido? Se forem mais de um, quais são?

6- Qual alimento você não gosta? Se for mais de um, quais são?

7- Quanto aguçado é o seu paladar? Assinale a alternativa:

\begin{tabular}{|l|l|}
\hline & PALADAR \\
\hline EXCELENTE & \\
\hline BOM & \\
\hline REGULAR & \\
\hline
\end{tabular}

Muito Obrigada!!

A sua colaboração foi muito importante!!! 


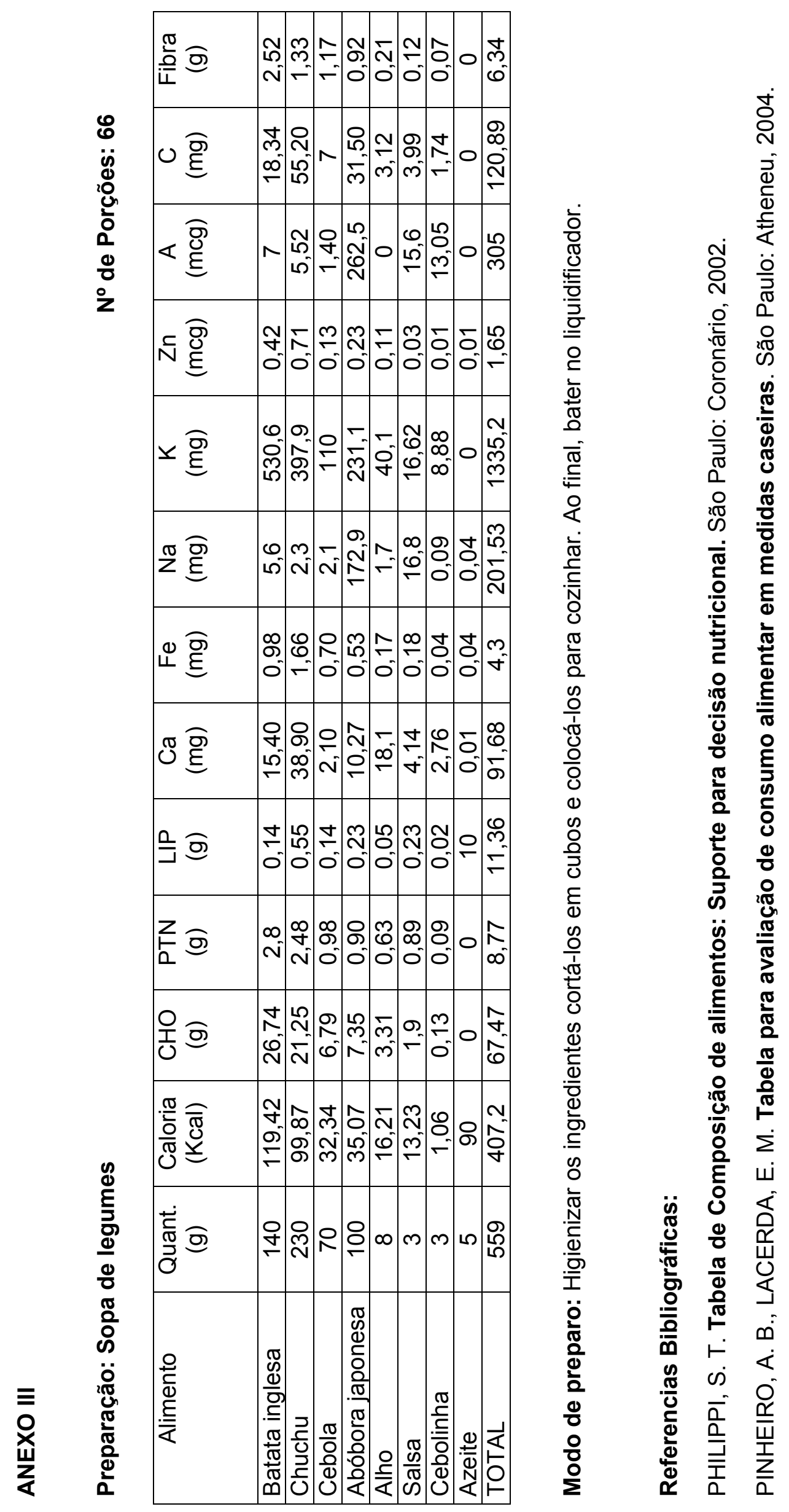




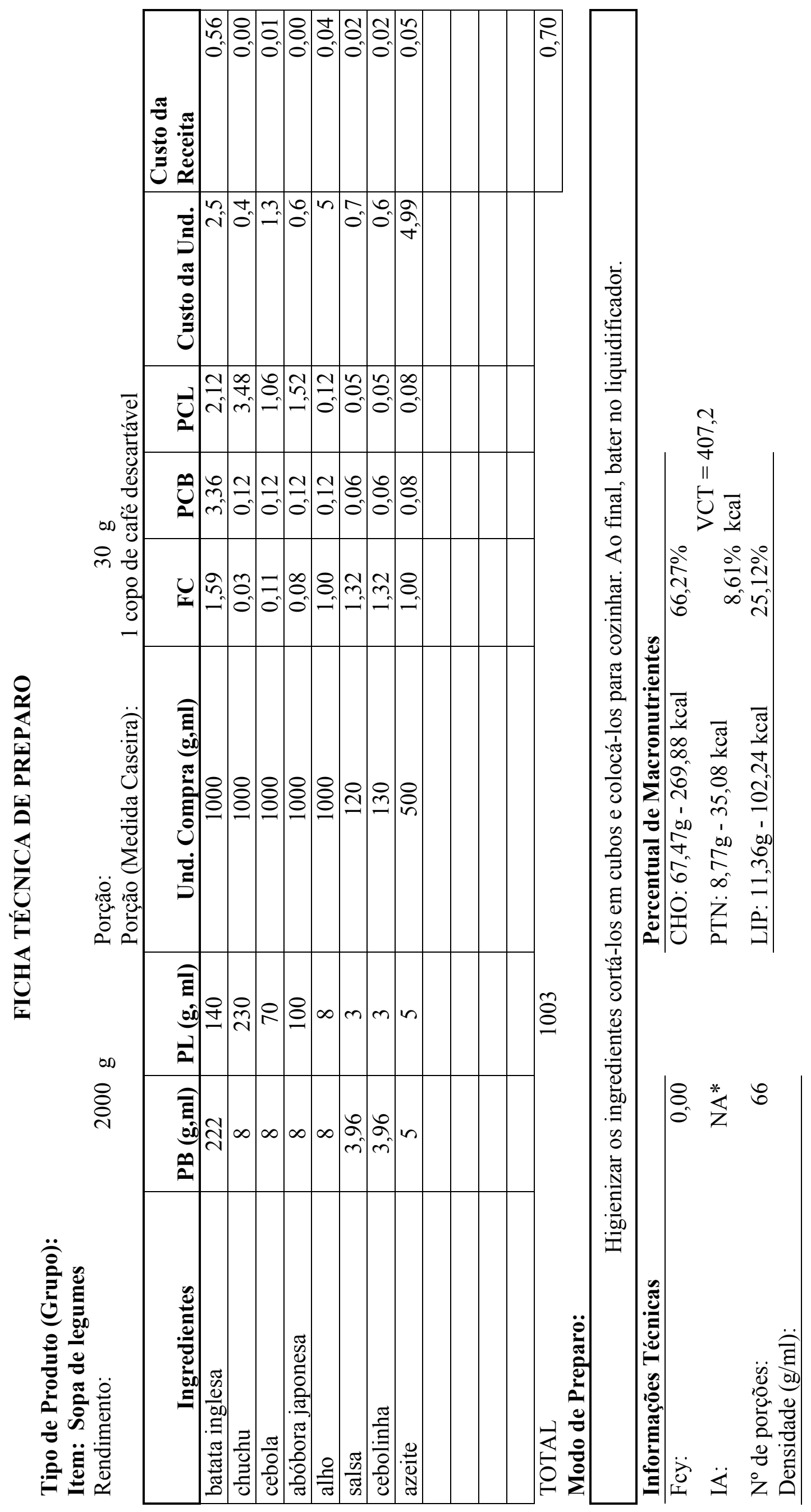


